\title{
A female patient with painful abdominal lump- torsion of wandering spleen
}

\section{${ }^{1}$ Rajendra $S$}

${ }^{1}$ Faculty of Medicine, Jaffna

\begin{abstract}
We present a 65 year old female patient presented with acute onset of severe left sided abdominal pain associated with a tender intra-abdominal lump over the left flank. An abdominal ultrasonography revealed an ectopic spleen in left flank and a CE-CT scan of abdomen confirmed the wandering spleen with torsion of vascular pedicle at the hilum. She underwent splenectomy. Wandering spleen is an uncommon clinical entity. Review of literature was made on different clinical presentations, diagnostic evaluation, treatment options, and clinical outcomes of patients with wandering spleen and discussed in this case report.
\end{abstract}

\section{Keywords}

wandering spleen, ectopic spleen, torsion, splenectomy, splenopexy

\section{Introduction}

Wandering spleen was first described by Van Horne as an autopsy finding in 1667. About 400 cases of wandering spleen have been reported around the world since then. Splenectomy for a wandering spleen was first carried out in Germany by an obstetrician, Dr.Martin, in 1877(1).

Two third of cases are seen in adults, usually in their $20-40$ years of age and up to $80 \%$ of adult cases are females. When it occurs in children less than 10 years of age both sexes are equally affected (2).

\section{Case report}

A 65-year-old mother of 6 children presented with severe abdominal pain over left flank for three days. She had intermittent vague central abdominal pain for the last three years. These episodes of pain lasted for few hours and subsided spontaneously. She did not have associated nausea, vomiting or lump at umbilicus. The current episode of abdominal pain was severe in nature and was associated with nausea and vomiting but she had normal bowel opening. She had mild fever and had a vague lumpiness over the left abdomen. She had hypertension and was not allergic to medicine or plaster. On examination her pulse rate was 110/ minute and blood pressure was $140 / 70 \mathrm{mmHg}$. There was a tender intrabdominal lump over the left flank (Fig.1).

Figure 1. Wandering spleen pre-operative marking and intra- operative appearance

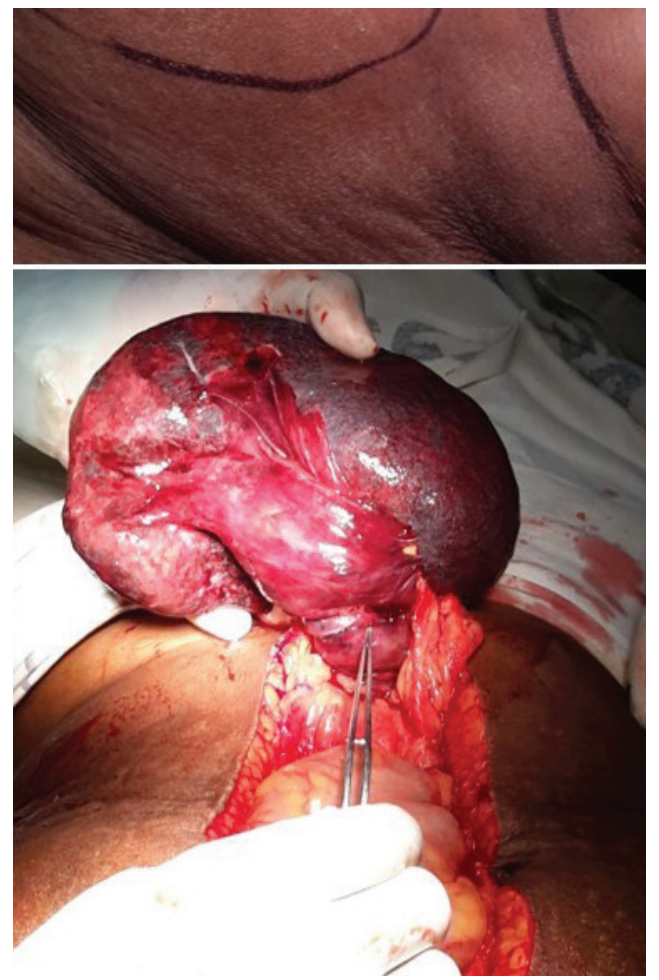

Corresponding Author: Rajenthra S. Email: dr.s.rajendra@gmail.com, (D) https://orcid.org/0000-0002-3303-603X, , Submitted April 2021 Accepted June2021

This is an open-access article distributed under the terms of the Creative Commons Attribution 4.0 International License, which permits unrestricted use, distribution and reproduction in any medium provided the original author and source are credited 
Figure 2. Wandering spleen CT - Angiogram appearance showing whirl appearance and lack of arterial flow

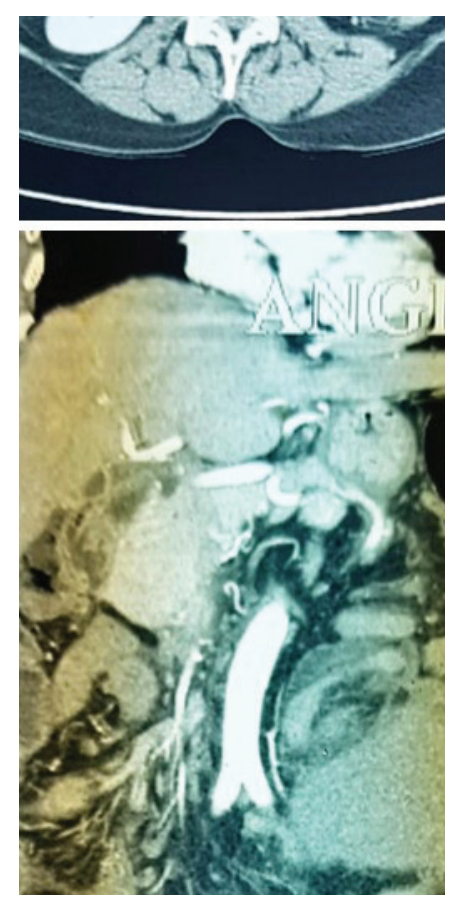

Digital rectal examination was normal. An ultrasound scan (USS) examination performed in her reveled absence of spleen in left hypochondrium and it was found in left lower abdomen close to midline with no demonstrable blood flow in the splenic hilum. The CT angiogram demonstrated an ectopic spleen with torsion of vascular pedicle at the hilum characterized by "whirl" sign. Spleen showed very mild enhancement favoring reduction in blood flow (Fig.2). After the preoperative optimization a laparotomy was performed on her. The spleen was found in the left flank adhering to greater omentum and intestine. The splenic pedicle was found to be twisted clockwise by $720^{\circ}$ (Fig. 1). Spleen was brought to the surface by adhesioloysis and detorsion was carried out in counterclockwise manner. The spleen was found non- viable, and a splenectomy was performed. She had an uneventful post operative recovery. She was given vaccines against Haemophilus influenzae, Pneumococcus pneumonia and Neisseria meningitides before being discharged from hospital.

\section{Discussion:}

Congenital absence of primary ligaments or malformations of the normal splenic suspensory ligaments cause the spleen to drop into lower abdomen from left hypochondrium. Wandering spleen is also known as ectopic spleen. Acquired conditions, such as connective tissue disease, splenomegaly and multiple pregnancies, cause laxity of these suspensory ligaments. Multiple pregnancies would have weakened the suspensory ligaments in this patient. When it happens spleen starts to "wander" in the abdominal cavity with its elongated vascular pedicle. When it moves, the vascular pedicle can get twisted leading to ischemia and infarction of spleen $(3,4)$.

Patients with wandering spleen maybe asymptomatic and it can be detected incidentally by imaging for other conditions. They can also present with a moving abdominal lump. They also can have abdominal pain when the vascular pedicle twists causing engorged spleen, splenic capsular stretch and localized peritonitis. Body movements, intestinal motility, respiratory movements and distension of adjacent organs can cause torsion at the splenic vascular pedicle. The type of pain varies depending on the degree of vascular occlusion. So the patients can experience chronic, intermittent abdominal or pelvic pain and discomfort due to recurrent torsion and detorsion of splenic pedicle. This patient had similar kind of intermittent abdominal pain before presenting with acute abdomen. Acute severe abdominal pain associated with fever and vomiting results from acute torsion of splenic pedicle. Acute torsion can be complicated with gangrene and abscess formation in spleen. It can also cause intestinal obstruction, gastric compression or pancreatitis due to necrosis of tail of pancreas $(2,4)$. 
Physical examination of abdominal may detect a lower abdominal mass. This patient had a tender mass in left flank close to midline (Fig.1). It is difficult to make a diagnosis of wandering spleen based on history and examination because these are non specific. Imaging of abdomen is important to make a diagnosis (5). USS assessment is widely used first line investigation to make a diagnosis of wandering spleen. It has 55\% accuracy rate. This investigation can detect absence of spleen in left hypochondrium and can demonstrate ectopic spleen, usually lying in left mid abdomen, based on sonographic morphology and echotexture. Colour duplex ultrasonography can assess the blood flow characteristics in splenic vascular pedicle when torsion is suspected. Contrast enhanced CT scan can demonstrate an acute torsion as alternate radiodense and radiolucent circular bands at the hilum of spleen. This "whirl sign" is specific for torsion of splenic pedicle and it may mimic a bowel intussusception. An intravenous contrast will not demonstrate enhancement of spleen if torsion had occurred. The CT angiogram in this patient clearly demonstrated the ectopic spleen with torsion of vascular pedicle (Fig. 2). The CT imaging can also give information about pancreatic tail necrosis and ascites $(2,6)$. When the patient presents with acute abdomen, the diagnosis of wandering spleen would be sometimes made at emergency laparotomy (2).

Surgery is the treatment of choice for wandering spleen. Conservative management is not recommended even for asymptomatic wandering spleen because $65 \%$ of them can develop complications (2).

Splenopexy and splenectomy are the surgical options to manage wandering spleen. The decision about the type of surgical intervention is dependent on the timing of presentation of wandering spleen and the viability of ectopic spleen during surgery. Both splenopexy and splenectomy, can be done by Vol.33, No.1, July 2021 either open surgical or laparoscopic methods $(2,7)$.

Splenopexy is the preferred surgical option for the non infarcted wandering spleen. Splenopexy can be done by wrapping it with an absorbable mesh and suturing the mesh on to stomach, diaphragm and abdominal wall. It can also be carried out, creating a pocket by mobilizing the peritoneum over the left posterolateral abdominal wall (over the diaphragm and muscle) and placing the spleen in the created space. The peritoneal flap is snugly sutured to stomach and colon with $4 / 0$ prolene. Omentum is brought over this area to prevent bowel adhesions. Partial splenectomy and splenopexy has also been described in cases with partial infarction of spleen. Splenopexy avoids the possibility of post splenectomy sepsis (7).

When the spleen appears to be infarcted at laparotomy or at laparoscopy, then splenectomy will be performed. The vaccines against Haemophilus influenzae, Pneumococcus pneumonia and

Neisseria meningitides will have to be given to the patient in the post operative period. This reduces the risk of overwhelming post splenectomy infection.

\section{Learning points}

Clinical presentation of wandering spleen varies from incidental finding to presentation with acute abdomen. It is a rare clinical condition and the diagnosis is usually made with the help of imaging. Splenopexy and splenectomy are the treatment options for wandering spleen.

\section{References:}

1. Magowska A. Wandering spleen: a medical enigma, its natural history and rationalization. World journal of surgery. 2013 Mar 1;37(3):54550. DOI: $10.1007 / \mathrm{s} 00268-012-1880-\mathrm{x}$

2. Dahiya N, Karthikeyan D, Vijay S, Kumar T, Vaid M. Wandering spleen: Unusual presentation and course of events. Indian 
Journal of Radiology and Imaging. 2002 Aug 1;12(3):359.

3. Available from: http://www.ijri.org/text. asp?2002/12/3/359/28483 Blouhos K, Boulas KA, Salpigktidis I, Barettas N, Hatzigeorgiadis A. Ectopic spleen: An easily identifiable but commonly undiagnosed entity until manifestation of complications. International journal of surgery case reports. 2014 Jan 1;5(8):451-4. DOI: 10.1016/j.ijscr.2014.05.01

4. Sodhi KS, Gupta P, Rao KL, Marwaha RK, Khandelwal N. Marfanoid hypermobility syndrome and skeletal abnormalities in a rare case of torsion of wandering spleen. The British journal of radiology. 2008 May;81(965):e145-8. DOI: $10.1259 / \mathrm{bjr} / 30123041$
5. FonsecaAZ, Ribeiro Jr M, Contrucci O. Torsion of a wandering spleen treated with partial splenectomy and splenopexy. The Journal of emergency medicine. 2013 Jan 1;44(1):e33-6. DOI: 10.1016/j.jemermed.2011.06.146

6. Benevento A, Boni L, Dionigi G, Ferrari A, Dionigi R. Emergency laparoscopic splenectomy for" wandering"(pelvic) spleen. Surgical endoscopy. 2002 Sep 1;16(9):1364c. DOI:10.1007/s00464-002-4213-6

7. Soleimani M, Mehrabi A, Kashfi A, Fonouni H, Buchler MW, Kraus TW. Surgical treatment of patients with wandering spleen: report of six cases with review of the literature. Surg Today 2007; 37:261-9. DOI: 10.1007/s00595-0063389-0 